\title{
Laboratory leaching tests to investigate mobilisation of metals within engineered geothermal reservoirs
}

\author{
Christopher Rochelle ${ }^{1, *}$, Andrew Kilpatrick ${ }^{1}$, Máté Osvald ${ }^{2}$, János Szanyi ${ }^{2}$, Tamás \\ Medgyes $^{2}$, and Balázs Kóbor ${ }^{2}$ \\ ${ }^{1}$ British Geological Survey, Nicker Hill, Keyworth, Nottingham, NG12 5GG, UK \\ ${ }^{2}$ University of Szeged, Department of Mineralogy, Geochemistry and Petrology, \\ Egyetem u. 2. Szeged H-6722
}

\begin{abstract}
Combining geothermal energy utilization with the extraction of metals in a single interlinked process offers a way to improve the economics of engineered geothermal systems. Here we describe laboratory experiments used to assess the effectiveness of a range of leaching fluids by quantifying metal release from various mineralised rocks. The main findings of this study include: enhanced mobilisation of metals typically found in sulphide minerals $(\mathrm{Pb}, \mathrm{Zn}, \mathrm{Cu})$, lesser mobilisation of some critical elements (such as $\mathrm{Co}, \mathrm{Sr}$ and $\mathrm{W}$ ), and the efficacy of organic additives in mobilising metals.
\end{abstract}

\section{Introduction}

The idea of using geothermal brines for mineral extraction has existed for decades, with lithium currently being an element of interest $[1,2,3]$. However, a wide spectrum of other elements may also be suitable for extraction [4,5]. Here we consider engineered geothermal systems (EGS), where recirculation of hot fluids would facilitate the extraction of dissolved metals, as well as energy, in surface plant. This would generate a second source of revenue, and thus increase the economic attractiveness of EGS development. This concept is the focus of the project 'Combined Heat, Power and Metal extraction from ultradeep ore bodies' (CHPM2030 ${ }^{3}$ ), where the potential for exploiting hot metal-bearing geological formations at depths greater than $3 \mathrm{~km}$ is being investigated. The strategic objective of the CHPM2030 project is to develop a novel technological solution to both make geothermal energy more attractive and to reduce Europe's dependence on the import of metals and fossil fuels [6].

In the envisioned technology, an EGS is established within a metal-bearing geological formation at depths greater than $3 \mathrm{~km}$ (Figure 1). A key aspect of such a scheme is that sufficient quantities of metals can be mobilised and transported to make the process economic, and critical factors underpinning this are the rates and magnitudes of metal release. Laboratory experiments simulating in-situ conditions, using batch or flow-through

\footnotetext{
* Corresponding author: caro@bgs.ac.uk

* https://www.chpm2030.eu
} 
reactors, are a useful way to provide well-constrained data to help to understand these. Such experiments also allow testing of different fluid compositions in order to ascertain if there are specific additives that may improve the metal recovery process [7]. Furthermore, evidence for the degree of leaching may allow assessment of whether it might increase system performance over time - through, for example, silicate and ore mineral dissolution and consequent permeability enhancement.

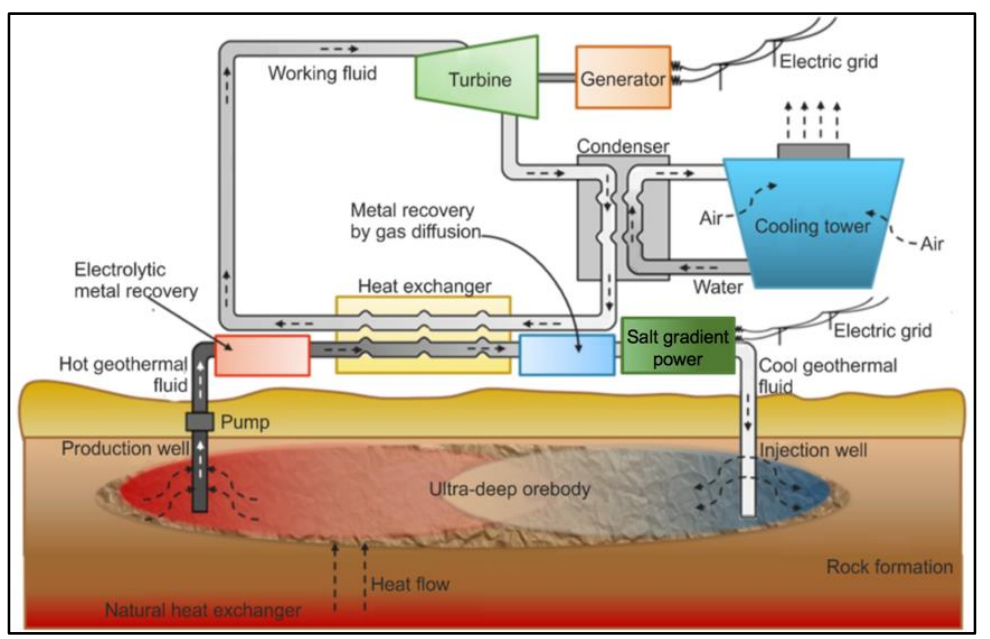

Fig. 1. Schematic representation of the CHPM concept. The information presented here relates to the release of metals from the 'ultra-deep orebody' and into the recirculating geothermal fluid.

\section{Methods}

Initial lower temperature experiments (presented in [7, 8]) explored the leaching potential of various fluids, and those showing more promise were used to leach a wider range of mineralised samples under higher pressure/temperature conditions typical of those that might be expected at 3-4 km depth. Here we present results using rock samples reacted with fluids that were very benign (deionised water), to slightly aggressive $(0.1 \mathrm{M}$ acetic acid), to relatively aggressive (dilute mineral acid: mixed $0.01 \mathrm{M}$ hydrochloric acid and $0.003 \mathrm{M}$ nitric acid). Relatively simple solutions were used in our tests as we were studying the comparative behaviour of metals. However, the authors recognise that the chemical composition (and especially salinity) of in-situ groundwater will be important, though there is a general lack of information on the chemistry of deep groundwaters. While mineral acids, such as nitric or hydrochloric acid, clearly have more potential to dissolve rock and associated mineralisation, dissolution potential is not the only consideration. Also of importance is the potential impact on the reservoir, geothermal plant infrastructure, and the wider environment. There is thus a trade-off between these different aspects, and hence it is useful to investigate and compare the relative performance of a variety of potential leachants.

\subsection{Materials}

In the work reported here, a sample of metamorphosed sediments with partial quartz veining, was used. It is a mixed sample from material representative of quartz vein mineralisation (containing $87 \%$ quartz, $5 \%$ muscovite, $2 \%$ dolomite, $5 \%$ galena, minor albite, chlorite, pyrite, and sphalerite) found at Herodsfoot, Cornwall, UK. The mixture was 
used to provide a more representative 'bulk' mineralogy for use in experiments. We also prepared samples from Rudabánya and Recsk (Hungary) and from Masca-Cacova Ierii (Romania), but this is reported elsewhere [7, 8]. The sample was crushed, sieved, and cleaned to produce a $500-250 \mu \mathrm{m}$ fraction free of fines, which was used for the experimental and analytical work. This fraction was cleaned, to remove fines and surface impurities, by repeated rinsing in acetone, until the supernatant ran clear, and was then oven dried at $30^{\circ} \mathrm{C}$.

\subsection{Experimental methods}

Here we focus on experiments at higher temperatures (a summary of the lower temperature experiments is given elsewhere [8]).

Batch experiments: Batch experiments were conducted at $100-200^{\circ} \mathrm{C}$ and under 200 250 bar pressure in titanium reactors inside thermostatically-controlled, fan-assisted ovens $[9,10,11]$. Into each was added $8.75 \mathrm{~g}$ of granulated rock and $350 \mathrm{ml}$ of leaching solution, plus a magnetic stirrer bead. Fluids were sampled via a titanium dip tube. To minimise mechanical damage to the solid, a caged stirrer bead was used, and only activated for 2 minutes every 4 hours. Nitrogen gas was used to pressurise the experiments. At the end of each experiment, as much of the solution as possible was removed prior to cooling. Once well below $100{ }^{\circ} \mathrm{C}$, the vessel was slowly depressurised, dismantled, and reacted rock grains recovered for subsequent analysis. Experiments ran for 600-1000 hours.

Flow-through experiments: Leaching processes were also investigated under continuous flow conditions using a stainless steel HPLC column reactor $(250 \mathrm{~mm}$ long, $21.2 \mathrm{~mm}$ inner diameter) containing $150 \mathrm{~g}$ of granulated rock sample. Pressure inside the column was maintained by an Econ Kappa 10 HPLC pump and a back-pressure regulator fitted at the outflow of the column. A digital thermostat allowed heating bands attached to the column to maintain temperatures to $\pm 1{ }^{\circ} \mathrm{C}$. Leaching tests operated at $200-250{ }^{\circ} \mathrm{C}$ and 250 bar. Flow was $0.5 \mathrm{ml}$ per minute, giving a residence time of 30-50 minutes, with an experimental duration of 8 hours.

\section{Results and discussion}

Figure 2 shows the sum of mobilised 'common' elements from the Cornwall sample at approximately $80 \mathrm{ppm}, 300 \mathrm{ppm}$ and 213,000 ppm for leaching experiments using deionised water, $0.1 \mathrm{M}$ acetic acid and $0.13 \mathrm{M}$ mineral acid (a mixture of $0.01 \mathrm{M}$ hydrochloric acid and $0.003 \mathrm{M}$ nitric acid) respectively. The large increase in total dissolved elements in the latter is largely due to silica (approximately 211,000 ppm) from the dissolution of silicate minerals. Whilst the dissolution of these may enhance flow paths in the geothermal reservoir through increased permeability, there is potential for it to also enhance mineral precipitation in surface infrastructure during the depressurisation and cooling of the geothermal fluids.

In terms of 'traditional' ore metals, there were notable increases in dissolved $\mathrm{Ni}, \mathrm{Pb}$, and $\mathrm{Zn}$ as more acidic solutions were used. These (together with the increases in $\mathrm{Fe}$ ) suggest sulphide mineral dissolution. Leaching was most rapid in the first few 10s of hours for both types of experiments, and in the batch experiments slowed considerably after several hundred hours of reaction. Relatively fast reactions would benefit the CHPM concept, as they would maximise metal concentrations in solution even with relatively short fluid residence times in the geothermal reservoir. However, on a reservoir scale this would mean that a formation with limited 'ore minerals' will deplete over time, and thus metal extraction would be most efficient early on in an extraction project, with returns likely to decrease on the medium to long term [12]. It is thus important to understand how leaching rates will vary over time. 
Total concentrations of 'at risk elements' (elements which, due to value or scarcity, are relatively desirable) are approximately $50 \mathrm{ppb}, 830 \mathrm{ppb}$, and $8500 \mathrm{ppb}$ for leaching using deionised water, $0.1 \mathrm{M}$ acetic acid and $0.13 \mathrm{M}$ mineral acid (the mixture of $\mathrm{HCl}$ and $\mathrm{HNO}_{3}$ ) respectively (Figure 3). Again, there is a strong relationship between acidity and the total amount of mobilised elements. The results indicate, however, that even a relatively mild leachant, such as acetic acid, with a $\mathrm{pH}$ of around 2.8 , can significantly increase leaching potential. In this case, a switch from deionised water to acetic acid generated a 20 -fold increase in dissolved load of the selected 'at risk' elements.

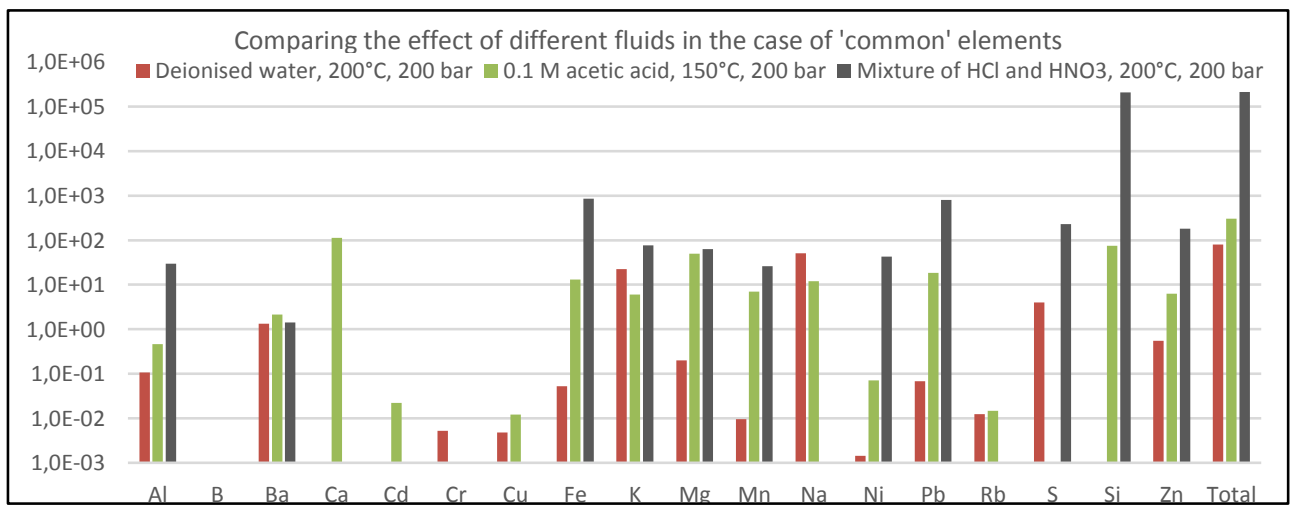

Fig. 2. Comparison of the effectiveness of different fluids for 'common' elements, concentrations are in ppm.

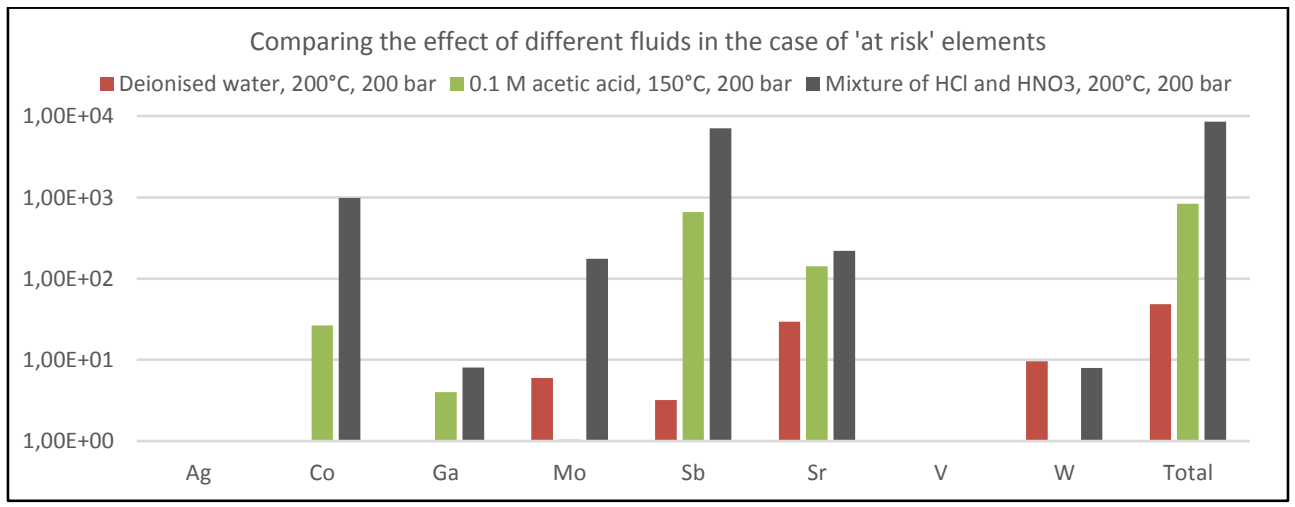

Fig. 3. Comparison of the effectiveness of different fluids for a selection of 'at risk' elements concentration are in ppb.

\section{Conclusions}

Recovery of metals from recirculating fluids has been proposed as a way to improve the economics of operating engineered geothermal systems. Fundamental to this is an understanding of metal release processes, and we have conducted preliminary batch and flow-through laboratory leaching tests at up to $250{ }^{\circ} \mathrm{C}$ and 300 bar to investigate the potential for metal mobilisation under in-situ conditions. We report tests on mineralised rocks from Cornwall (UK), which were reacted with a range of leaching fluids, including deionised water, dilute acetic acid, and dilute mineral acid (the mixture of $\mathrm{HCl}$ and $\mathrm{HNO}_{3}$ ), for up to 1000 hours. 
Increases were found in the concentration of many metals, and were broadly correlated with increasing temperature and acidity. It was also noted that the presence of suitable ligands also increased metal concentration in solution, this included $\mathrm{Cl}^{-}$and especially organics (e.g. acetic acid). Detected elements were grouped as 'common' elements (less economic importance and higher occurrence), and 'at risk' (those having higher economic value defined by the European Union). One of the highest concentrations of 'common' elements was lead (up to $870 \mathrm{ppm}$ with acetic acid in batch experiments) and up to $540 \mathrm{ppm}$ in flow-through experiments. Notable concentrations of $\mathrm{Fe}$ and $\mathrm{Zn}$ are also present in leachates, due to enhanced dissolution of sulphide mineralisation in the samples.

Significant concentrations of $\mathrm{Al}$ and $\mathrm{Si}$ were also found in some leaching solutions, indicating considerable dissolution of matrix silicates, such as quartz and mica, present in the samples. This could be desirable in terms of increasing reservoir permeability and opening flow paths, but if concentrations become too high there is an increased risk of precipitation due to saturation with secondary phases, which could clog fractures and inhibit fluid flow in a geothermal reservoir, and risk fouling boreholes or surface infrastructure. Notable was that dilute acetic acid solutions achieved relatively high concentrations of some metals, but comparatively low concentrations of $\mathrm{Al}$ and $\mathrm{Si}$, so limiting the potential for the formation of potentially problematical precipitates. We recognise however, that full extrapolation to a natural setting will require consideration of groundwater chemistry, fluid migration pathways and residence times, variations in surface areas, and decreases in available metals over time, and ongoing work seeks to investigate this. The data gathered here suggests that, given sufficient characterisation of the mineralogy and fluid chemistry of deep geothermal systems, it may be possible to tailor the chemistry of leaching solutions to maximise metal recovery.

Acknowledgement. This work was supported by the European Union's Horizon 2020 research and innovation programme under grant agreement No. 654100 (CHPM2030 project). C. Rochelle and A. Kilpatrick publish with the permission of the Executive Director of the British Geological Survey, UKRI.

\section{References}

1. V.D. Dang \& M. Steinberg, Energy, 3(3), 325-336 (1978)

2. W.P.C. Duyvesteyn, Geothermics, 21(5-6), 773-779 (October-December 1992)

3. S.E. Kesler, P.W. Gruber, P.A. Medina, G.A. Keoleian, M.P. Everson \& T.J. Wallington, T.J. Ore Geology Reviews, 48, 55-69 (2012).

4. R.G. Bloomquist, Geotherm. Resour. Council, 30, 579-582 (2006)

5. Neupane, G., Wendt, D. S. 2017: Proceedings of 42nd Workshop on Geothermal Reservoir Engineering Stanford University, Stanford, CA (2017)

6. European Commission DG for Internal Market, Industry, Entrepreneurship and SMEs; Study on the review of the list of Critical Raw Materials: Critical Raw Materials Factsheets. ISBN: 97892-79-72119-9, DOI: 10.2873/398823 (2017)

7. A. Kilpatrick, C. Rochelle, J. Rushton et al. Report on metal content mobilisation using mild leaching, CHPM2030 report D2.2, 374p. DOI: 10.5281/zenodo.1207069 (2017)

8. M. Osvald, A.D. Kilpatrick, C.A. Rochelle et al., Geofluids (to be published)

9. K. Bateman, C.A. Rochelle, G. Purser et al., Energy Procedia, 37, 5307-5314 (2013)

10. E.B. Moyce, C.A. Rochelle, K. Morris et al., Appl. Geochem., 50, 91-105 (2014)

11. C.A. Rochelle, A.E. Milodowski, K. Bateman, et al., Min. Mag., 80(6), 1089-1113 (2016)

12. J. Szanyi, M. Osvald, T. Medgyes et al., Recommendations for Integrated Reservoir Management. CHPM2030 report D2.1, 119p. DOI: 10.5281/zenodo.1204833 (2017) 